\title{
Recurrent Prosthetic Knee Infection Associated with a Hidden Nidus in the Quadriceps Tendon
}

\author{
Joao Pedro Gouveia Nobrega ${ }^{1}$, Fabio Sousa ${ }^{2 *}$, Carla Brazao ${ }^{3}$, Joao Pereira ${ }^{4}$, Paulo Dourado ${ }^{5}$
}

\author{
1,3,4 Orthopaedic surgeon resident Hospital Espirito Santo de Evora, EPE \\ ${ }^{2}$ Orthopaedic surgeon resident Hospital Fernando Fonseca \\ ${ }^{5}$ Orthopaedic surgeon Hospital Espirito Santo de Evora
}

DOI: $10.36347 /$ sasjm.2022.v08i01.007

| Received: 16.12.2021 | Accepted: 19.01.2022 | Published: 23.01.2022

*Corresponding author: Joao Pedro Gouveia Nobrega

Orthopaedic surgeon resident Hospital Espirito Santo de Evora, EPE

\section{Abstract}

We present a case of a 60-year-old male with poorly controlled type 2 Diabetes that had recurrent prosthetic knee infection that besides antibiotic or surgical treatment was difficult to treat. On the latest revision, he presented with an infection of the knee spacer with severe stiffness impairing knee flexion. In the debridement phase, we noticed a nidus of purulent tissue on the quadriceps tendon hidden inside an apparently normal peritendon. This case highlights several management details to avoid recurrent catastrophic prosthetic knee infection recurrence such as: the importance of perioperative management of comorbid diseases such as hyperglycaemia and poorly controlled diabetes that are a well-defined evidence-based risk factor for prosthetic joint infection and also the importance of adequate debridement as a balanced surgical gesture between excising too little or too much not to damage the soft tissue envelope.

Keywords: Prosthetic Knee Infection, Quadriceps tendon, recurrent, infection, knee arthroplasty, Diabetes. Copyright $(\mathcal{C} 2022$ The Author(s): This is an open-access article distributed under the terms of the Creative Commons Attribution 4.0 International License (CC BY-NC 4.0) which permits unrestricted use, distribution, and reproduction in any medium for non-commercial use provided the original author and source are credited.

\section{DESCRIPTION}

A 60-year-old male with type 2 Diabetes presented with pain, stiffness and periwound erythema three weeks after primary knee arthroplasty.

He was initially treated with empirical antibiotic therapy with co-amoxiclav however, the symptoms worsened and evolved to a pus-discharging sinus with positive cultures for Methicillin-Susceptible Staphylococcus Aureus (MSSA). A two-stage revision arthroplasty was performed with a total of 12 weeks of antibiotic therapy (flucloxacillin after explantation and rifampicin and levofloxacin after implantation).

One month after the prosthesis implantation, the patient was re-admitted with surgical wound dehiscence and purulent drainage positive for $\mathrm{S}$. dysgalactae. As a result, the patient underwent the first stage of the revision with implantation of a spacer and a reconstructive skin graft to cover the anterior tibial defect of the surgical wound. During this period, the patient had poorly controlled glycemic values with $\mathrm{HbA} 1 \mathrm{c}$ of $8.6 \%$.

Five months later, he presented again with pusdischarging sinus positive for $\mathrm{S}$. epidermidis and infection of the knee spacer was diagnosed.

A revision of the spacer was performed with an inverted "V" quadricepsplasty approach because of the stiffness impairing knee flexion. We noticed a suspicious devitalized tissue on a patella corner that communicate with the space inside peritenon of the quadriceps tendon. Therefore, a longitudinal incision on an apparently normal peritenon was performed and a large amount of purulent tissue within the fibers of the quadriceps tendon was found, with a partially disrupted tendon (Figure 1). Extensive debridment was performed and a gentamicin with clindamycin spacer was implanted. 


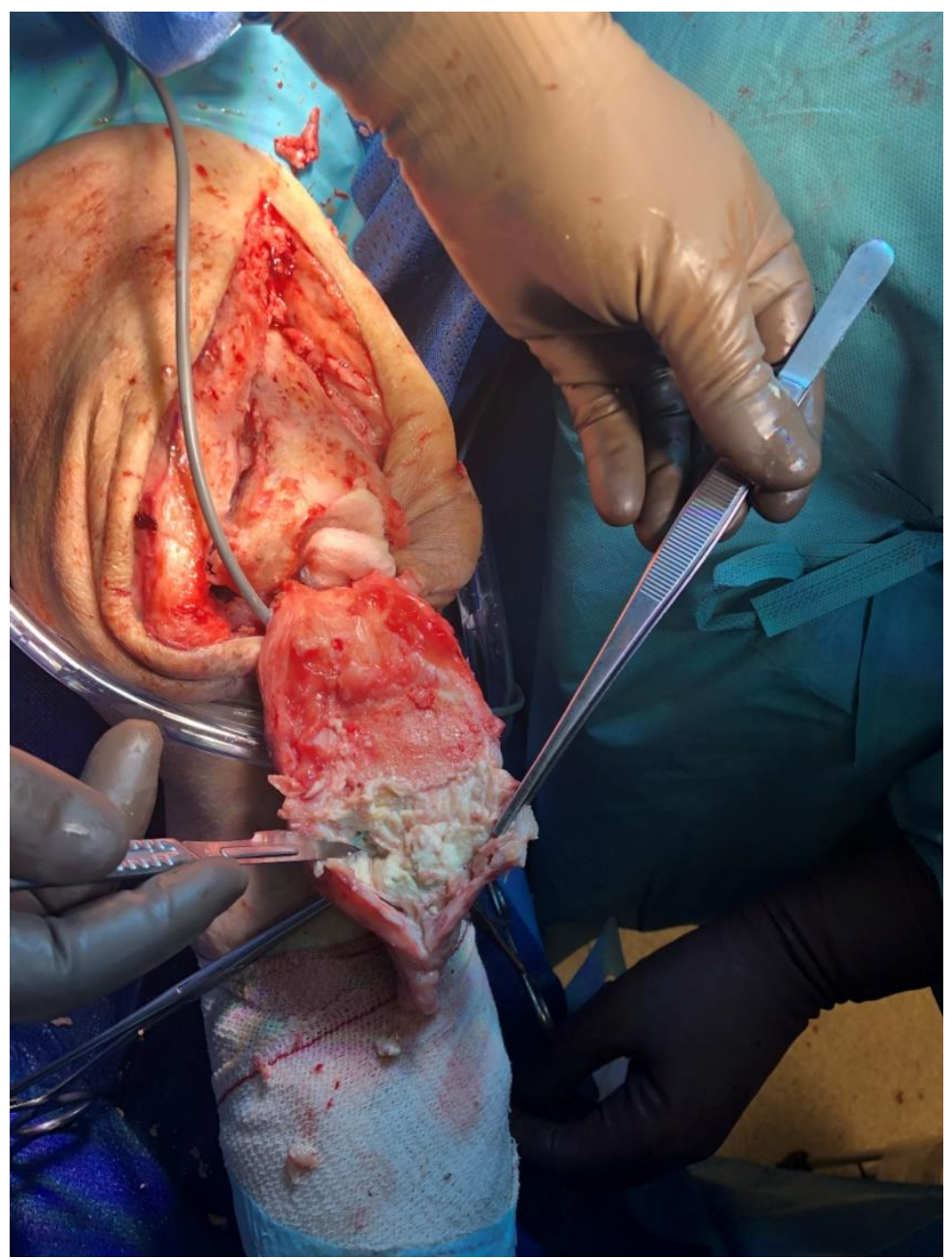

Figure 1: Infection nidus inside the healthy paratenon with the fibers of the quadriceps tendon partially disrupted. Note the abnormal thickened paratenon

The patient had an uneventful recovery with no evidence of recurrence of infection.

We believe that this bacterial nidus, hidden on an apparently healthy paratenon, together with the uncontrolled Diabetes and wound dehiscence contributed to the prosthetic joint infection (PJI) recurrence by different organisms.

This case highlights several aspects that clinicians should take into consideration to minimize the risk of PJI:

- The perioperative management of comorbid diseases is imperative to optimize patients conditions; It is recommended for diabetic patients to have glucose levels $4.4-10 \mathrm{mmol} / \mathrm{L}$ or/and HbA1C < 7.5\%; Glucose level > $11.1 \mathrm{mmol} / \mathrm{L}$ doubles the risk for PJI $[1,2]$.

- Another mistake, is the delay of treatment of acute PJI. On acute PJI, patient could be eligible for a one-stage procedure with less comorbidity [3]. Instead it was assumed as a superficial soft tissue infection.

- The scenario of a recurrent PJI demands a high index of suspicion for detection of atypical organisms (such as mycobacterium and fungal agents) [4] but also for polymicrobial PJI (being more likely to be infected with enterococcal species, E. coli, and gram-negative organisms [5] which should be considered when administering antibiotics to these patients.

- In the scenario of a polymicrobial PJI, it is possible that some organisms may have escaped isolation by culture and hence were not covered by the administered antibiotics.

- The importance of adequate debridement - any residual infected tissue left can lead to persistence of the infection but, it should be done carefully because extensive soft tissue dissection devascularizes bone, predisposing to further infection [6].

\section{LEARNING POINTS/TAKE HOME MESSAGES}

- Glycaemic levels should be optimized preoperatively - Hyperglycaemia and poorly controlled diabetes is a well-defined evidence based risk factor for PJI.

- Radical debridement is crucial for the success of a PJI treatment -as a balanced surgical gesture between excising too much or too little it is 
Joao Pedro Gouveia Nobrega., SAS J Med, Jan, 2022; 8(1): 28-30

advisable to be done by experienced surgeons; In the scenario of recurrent PJI we should look for atypical agents and rule out a polymicrobial infection.

- Inadequate long-term antibiotic treatment electing drug-resistant micro-organisms and the excessive time with a spacer acting as a foreign body enhances risk for biofilm formation as time passes and are considered common management errors that could contribute for recurrent PJI.

\section{REFERENCES}

1. Jämsen, E., Nevalainen, P., Eskelinen, A., Huotari, K., Kalliovalkama, J., \& Moilanen, T. (2012). Obesity, diabetes, and preoperative hyperglycemia as predictors of periprosthetic joint infection: a single-center analysis of 7181 primary hip and knee replacements for osteoarthritis. JBJS, 94(14), e101.

2. Mraovic, B., Suh, D., Jacovides, C., \& Parvizi, J. (2011). Perioperative hyperglycemia and postoperative infection after lower limb arthroplasty. Journal of diabetes science and technology, 5(2), 412-418.

3. Bengtson, S., \& Knutson, K. (1991). The infected knee arthroplasty: a 6-year follow-up of 357 cases. Acta Orthopaedica Scandinavica, 62(4), 301-311.

4. Kim, D. H., Bae, K. C., Kim, D. W., \& Choi, B. C. (2020). Risk factors of uncontrolled periprosthetic knee joint infection after two-stage reimplantation. Knee Surgery \& Related Research, 32, 1-7.

5. Tan, T. L., Kheir, M. M., Tan, D. D., \& Parvizi, J. (2016). Polymicrobial periprosthetic joint infections: outcome of treatment and identification of risk factors. JBJS, 98(24), 2082-2088.

6. Li, C., Renz, N., Trampuz, A., \& Ojeda-Thies, C. (2020). Twenty common errors in the diagnosis and treatment of periprosthetic joint infection. International orthopaedics, 44(1), 3-14. 